Hadron Structure '15

International Journal of Modern Physics: Conference Series

Vol. 39 (2015) 1560106 (10 pages)

(C) The Author(s)

DOI: 10.1142/S2010194515601064

\title{
Search for BFKL-evolution manifestations at high energies
}

\author{
Victor T. Kim \\ Petersburg Nuclear Physics Institute, NRC KI \\ Gatchina, 188300, Russia \\ and \\ St.Petersburg Polytechnic University \\ St. Petersburg, 195251, Russia \\ victor.kim@cern.ch
}

Published 26 November 2015

\begin{abstract}
A brief overview of searches for BFKL-evolution manifestations at high energies is presented. Data comparison with the predictions of BFKL-evolution in the leading logarithmic (LL) and next-to-leading logarithmic (NLL) approximations are discussed.
\end{abstract}

Keywords: GLAPD; BFKL; jet production.

PACS Numbers: 12.38.Aw, 12.38.Cy, 12.38.Qk, 13.87.-a

\section{Introduction}

Quantum Chromodynamics (QCD) being an essential ingredient of the Standard Model is well tested in hard processes when transferred momentum is of the order of the total collision energy (Bjorken limit: $Q^{2} \sim s \rightarrow \infty$ ). The cornerstones of perturbative QCD at this hard kinematic regime (QCD-improved parton model): factorization of inclusive hard processes and the Gribov-Lipatov-Altarelli-ParisiDokshitzer (GLAPD) evolution equation ${ }^{1-5}$ provides a basis for the successful QCDimproved parton model. The factorization theorem for inclusive hard processes with hadron(s) ensures that the inclusive cross section factorizes into partonic subprocess cross section and parton distribution function(s). The GLAPD evolution equation governs the $\log Q^{2}$-dependence (at $Q^{2} \rightarrow \infty$ ) of the inclusive hard process crosssections at fixed scaling variable $x=Q^{2} / s$.

Another important kinematic domain at high-energy is expected to be described by the (Balitsky-Fadin-Kuraev-Lipatov) BFKL-evolution, ${ }^{6-9}$ in so called QCD Gribov-Regge limit, whereby at fixed $Q^{2} \gg \Lambda_{Q C D}^{2}, s \rightarrow \infty$. In the Gribov-Regge

This is an Open Access article published by World Scientific Publishing Company. It is distributed under the terms of the Creative Commons Attribution 3.0 (CC-BY) License. Further distribution of this work is permitted, provided the original work is properly cited. 
limit, the BFKL evolution in the leading logarithmic (LL) approximation governs $\log (1 / x)$ evolution (at $x \rightarrow 0$ ). Note that the BFKL-evolution in the nextto-leading logarithmic (NLL) approximation, ${ }^{10-13}$ unlike the ${ }^{2 L}$ BFKL $^{6-9}$ partly includes leading order (LO) GLAPD evolution with the running coupling constant, $\alpha_{S}\left(Q^{2}\right)=4 \pi / \beta_{0} \log \left(Q^{2} / \Lambda_{Q C D}^{2}\right)$.

Therefore, the LL BFKL and especially the NLL BFKL ${ }^{10-13}$ are anticipated to be important theoretical tools for exploring the high-energy limit of QCD. In particular, this importance arises since the highest eigenvalue, $\omega^{\text {max }}$, of the BFKL equation is related to the intercept of the Pomeron, which in turn governs the high-energy asymptotics of the total cross-sections: $\sigma \sim\left(s / s_{0}\right)^{\alpha_{I P}-1}=\left(s / s_{0}\right)^{\omega^{\max }}$, where the Regge parameter $s_{0}$ defines the approach to the asymptotic regime. The BFKL Pomeron intercept in the LL turns out to be rather large: $\alpha_{I P}-1=\omega_{L O}^{\max }=$ $12 \log 2\left(\alpha_{S} / \pi\right) \simeq 0.54$ for $\alpha_{S}=0.2$. The NLL corrections to the BFKL have been calculated in Refs. 10 and 11, but the results in the $\overline{\mathrm{MS}}$ scheme have a strong renormalization scale dependence. This scale ambiguity dependence is , in particularly, due to large running coupling corrections. Naive application of BLM approach ${ }^{14}$ for reduction of the scale ambiguity for NLL BFKL result in $\overline{\mathrm{MS}}$ scheme does not lead to a sensible result.

In Ref. 12 it has been formulated an approach based on the asymptotic conformal properties of theory generalizing the Brodsky-Lepage-Mackenzie (BLM) optimal scale setting procedure ${ }^{14}$ for non-Abelian cases, i.e., for the processes which contain in the leading order non-Abelian interactions. The BLM optimal scale setting resums the conformal-violating $\beta_{0}$-terms into the running coupling in all orders of perturbation theory, thus preserving the conformal properties of the theory. It turns out $^{12}$ that the BLM procedure ${ }^{14}$ within physical non-Abelian renormalization schemes $^{15,16}$ eliminates the renormalization scale ambiguity of the NLL BFKL. ${ }^{10,11}$ (Hereafter, we will call the generalized BLM approach of Ref. 12 by BFKLP).

Strictly speaking, the integral kernel ${ }^{10,11}$ of the BFKL equation at NLL is not conformally invariant and, hence, one should use a more accurate method for its solution. ${ }^{17}$ But in BFKLP approach, ${ }^{12}$ the dependence of the eigenvalue of the kernel from the gluon virtuality is extremely weak and, therefore, $\omega_{N L L}$ coincides basically with the eigenvalue. ${ }^{13}$

Therefore, one of the striking features of the NLL BFKL analysis by BFKLP ${ }^{12}$ is that the NLL value for the intercept of the BFKL Pomeron, improved by the generalized BLM procedure, has a very weak dependence on the gluon virtuality $Q^{2}: \alpha_{I P}-1=\omega_{N L L}^{\max } \simeq 0.13-0.18$ at $Q^{2}=1-100 \mathrm{GeV}^{2}$ (Fig. 1). This agrees with the conventional Regge theory where one expects universal intercept of the Pomeron without any $Q^{2}$-dependence. The minor $Q^{2}$-dependence obtained leads to approximate conformal invariance.

Note, that the BFKLP approach ${ }^{12}$ generalizes the BLM procedure ${ }^{14}$ for nonAbelian cases, when the processes contain non-Abelian contributions at the LO, while the recent approaches in Refs. 18-20 and 21-24 generalize it for higher orders. 


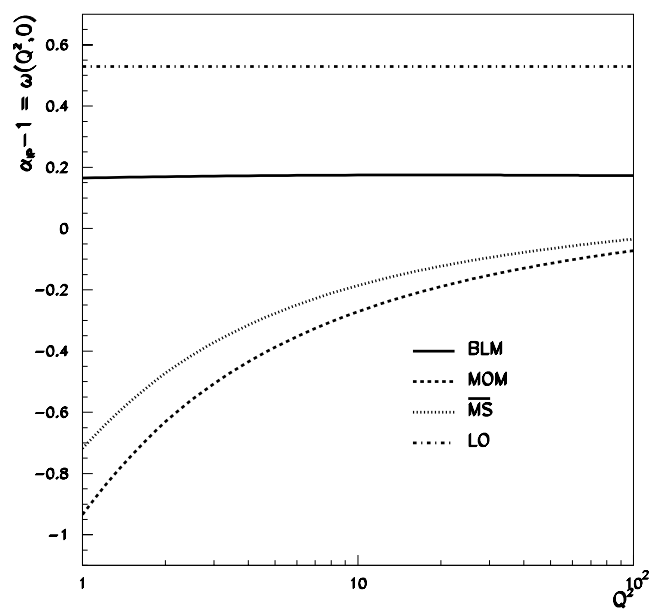

Fig. 1. The intercept of the BFKL Pomeron in NLL $\alpha_{I P}-1=\omega_{N L L}^{\max }$, improved by BFKLP, ${ }^{12}$ the generalized BLM approach for non-Abelian case.

It is important that BFKLP approach ${ }^{12}$ allows to use in the NLL BFKL many of celebrated features of LL BFKL, ${ }^{6-9}$ such as a conformal invariance, ${ }^{17}$ using the fact that a source of conformal invariance violation is the running coupling.

\section{BFKL for Photon-Photon Collisions}

As a phenomenological application of the NLL BFKL improved by BFKLP, ${ }^{12}$ the generalized BLM procedure, with its effective resummation of the conformalviolating $\beta_{0}$-terms into the running coupling in all orders of the perturbation theory, one can consider scattering highly virtual photons. ${ }^{25}$ The LL BFKL application for highly virtual photon scattering was considered in Refs. 26-30.

Photon-photon collisions, particularly $\gamma^{*} \gamma^{*}$ processes, play a special role in QCD, since their analysis is under much better control than the calculation of lepton-hadron and hadron-hadron processes, which require the input of nonperturbative hadronic structure functions or wave functions. In addition, unitarization (screening) corrections due to multiple Pomeron exchange should be less important for the scattering of highly virtual photons $\left(\gamma^{*}\right)$ than for hadronic collisions or real photon $(\gamma)$ scattering. ${ }^{31}$

The high-energy asymptotic behaviour of the $\gamma \gamma$ total cross section in QED can be calculated ${ }^{32-34}$ by an all-orders resummation of the leading logarithmic terms: $\sigma \sim \alpha^{4} s^{\omega}, \omega=\frac{11}{32} \pi \alpha^{2} \simeq 6 \times 10^{-5}$ (Fig. 2). However, the slowly rising asymptotic behaviour of the QED cross section is not apparent since large contributions come from other sources, such as the cut of the fermion-box contribution: $\sigma \sim \alpha^{2}(\log s) / s^{35}$ (which although subleading in energy dependence, dominates 


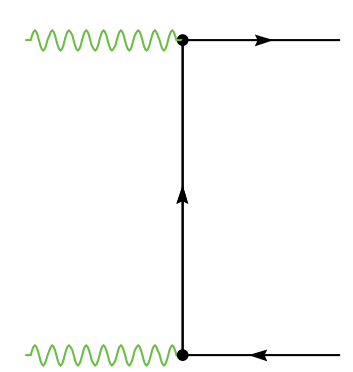

(a)

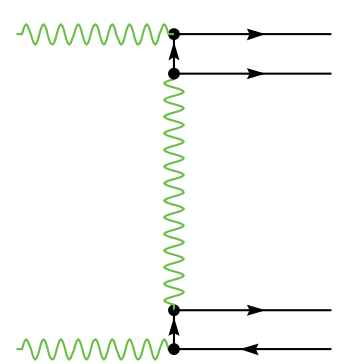

(b)

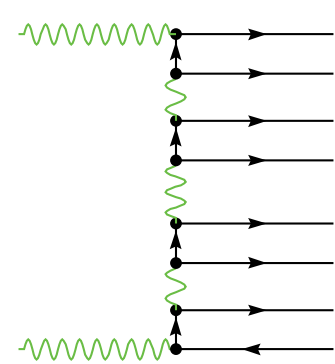

(c)

Fig. 2. Photon-Photon collisions in QED: (a) electron-box diagram: $\sigma \sim \alpha^{2}(\log s) / s$; (b) onephoton exchange diagram: $\sigma \sim \alpha^{4} s^{0}$; (c) a typical higher-order diagram; its resummation leads to $\sigma \sim \alpha^{4} s^{\omega}, \omega=\frac{11}{32} \pi \alpha^{2} .33$

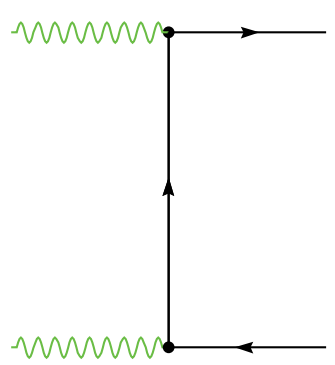

(a)

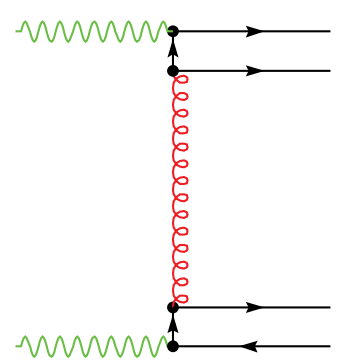

(b)

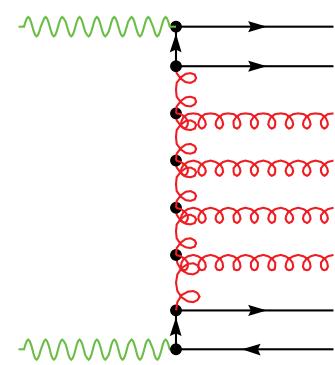

(c)

Fig. 3. High-energy photon-photon collisions in QCD: (a) quark-box diagram: $\sigma \sim \alpha^{2}(\log s) / s$; (b) one-gluon exchange diagram: $\sigma \sim \alpha^{2} \alpha_{S}^{2} s^{0}$; (c) a typical higher-order diagram; its resummation leads to $\sigma \sim \alpha^{2} \alpha_{S}^{2} s^{\omega}, \omega_{L L}=12 \ln 2\left(\alpha_{S} / \pi\right) \simeq 0.55^{6-9}$ and $\omega_{N L L}=0.13-0.18 .{ }^{12}$

the rising contributions by powers of the QED coupling constant) and QCD-driven processes (Fig. 3).

Figure 4 compares the LL and BLM scale-fixed NLL BFKL predictions $\sigma \sim$ $\alpha^{2} \alpha_{S}^{2} s^{\omega 25}$ with CERN LEP2 data from OPAL ${ }^{36}$ and L3. ${ }^{37}$ The spread in the curves reflects the uncertainty in the choice of the Gribov-Regge scale parameter, which defines the beginning of the asymptotic regime: $s_{0}=Q^{2}$ to $4 Q^{2}$ for LL and NLL BFKL, where $Q^{2}$ is the mean virtuality of the colliding photons. One can see from Fig. 4 that the agreement of the NLL BFKL predictions, ${ }^{25}$ improved by BFKLP ${ }^{12}$ with the data is quite good. The sensitivity of the NLL BFKL results ${ }^{25}$ to the Gribov-Regge parameter $s_{0}$ is much smaller than in the case of the LL BFKL. The variation of the predictions in the value of $s_{0}$ reflects uncertainties from uncalculated subleading terms. The parametric variation of the LL BFKL predictions is so large 


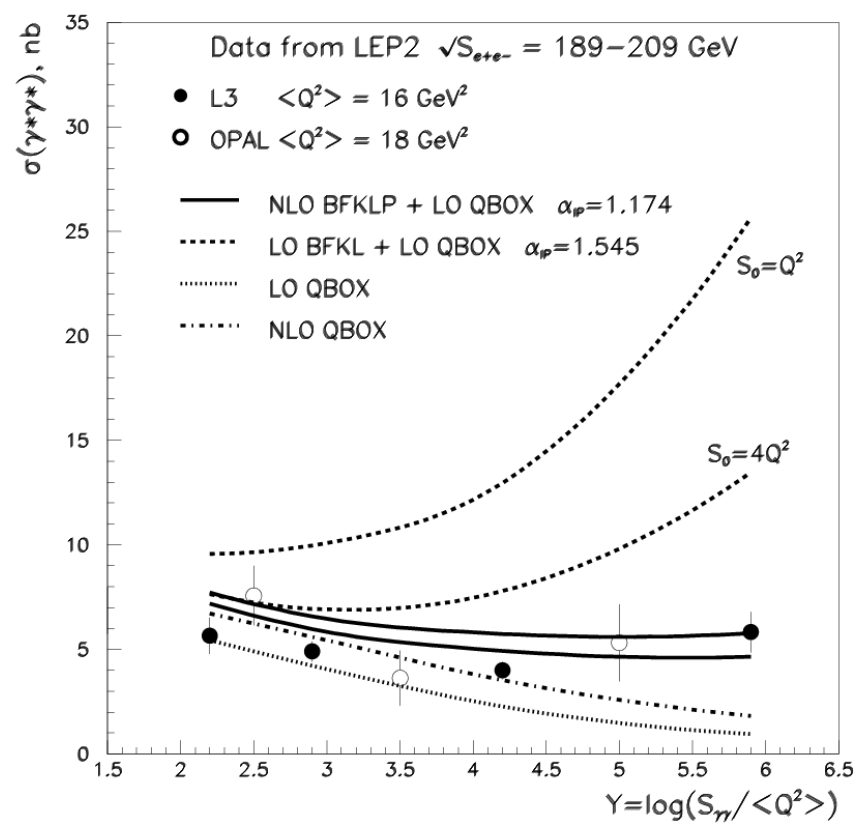

Fig. 4. The energy dependence of the total cross section for highly virtual photon-photon collisions predicted by the generalized BLM scale-fixed NLL BFKL ${ }^{25}$ compared with OPAL $^{36}$ and L $3^{37}$ data from LEP2 at CERN. The (solid) dashed curves correspond to the (N)LL BFKL predictions for two different choices of the Regge scale: $s_{0}=Q^{2}$ for upper curves and $s_{0}=4 Q^{2}$ for lower curves.

that it can be, in fact, neither ruled out nor confirmed at the energy range of CERN LEP2.

The next-to-leader order (NLO) impact factor of the virtual photon, which is needed for complete NLL BFKL calculations, has became recently known. ${ }^{38-40}$ However, it is still difficult to apply it for full NLL calculation of highly virtual photon cross section. So, currently one can use the leading order (LO) impact factor of Ref. 33, assuming that the main energy-dependent NLL corrections come from the NLL BFKL subprocess rather than from the photon impact factors. ${ }^{25,41}$

The double-logarithmic DGLAP asymptotics related with $\log \left(Q_{A}^{2} / Q_{B}^{2}\right)$-terms for the total photon-photon cross section was considered in Ref. 30 and found to be small for the CERN LEP2 kinematical region. The point is that most of the CERN LEP2 data ${ }^{36,37}$ are collected at the approximately equal virtualities of the colliding photons: $1 / 2<Q_{A}^{2} / Q_{B}^{2}<2$. It should be stressed that the soft Pomeron contribution to the $\gamma^{\star} \gamma^{\star}$ total cross section, if estimated within the vector-dominance model, is proportional to $\sigma_{\gamma^{\star} \gamma^{\star}} \sim\left(m_{V}^{2} / Q^{2}\right)^{4} \sigma_{\gamma \gamma}$ and therefore suppressed for such highly virtual photons as those under consideration.

We also note that to the NLL BFKL predictions ${ }^{25,41}$ improved by BFKLP ${ }^{12}$ should be added for consistency quark and anti-quark exchange contributions, ${ }^{42}$ 
which correspond to the secondary Reggeon exchange and should not play an important role at very high energies. On the other hand, at not very high energies such as at LEP2 it can yield a noticeable contribution. ${ }^{42}$

The NLO quark-box contribution, ${ }^{43}$ which is leading in coupling constant counting and subleading in energy dependence, underestimates the L3 data point at $Y \equiv \log \left(s_{\gamma \gamma} /\left\langle Q^{2}\right\rangle\right)=6$ by 4 standard deviations. Indeed, the NLO quark-box contribution, ${ }^{43}$ calculated in massless approximation, can be scaled down from general considerations with the quark masses. For example, at leading order, the inclusion of masses to the quark-box diagram reduces its contribution by $10-15 \% .{ }^{43}$ Also, the one-gluon exchange added to the $(\mathrm{N}) \mathrm{LO}$ quark-box contribution is not sufficient to describe the data at $Y=6$ within (3) 4 standard deviations.

To summarize this section, highly virtual photon-photon collisions provide a very unique opportunity to test high-energy asymptotic of QCD. The NLL BFKL predictions for the $\gamma^{*} \gamma^{*}$ total cross section improved by BFKLP approach ${ }^{12}$ show good agreement with the data from OPAL ${ }^{36}$ and L3 ${ }^{37}$ at CERN LEP2. Nevertheless, it will be interesting to compare the presented results with forthcoming complete NLL BFKL calculations for highly virtual photon collisions.

\section{BFKL Predictions for Jet Production in Hadron Collisions}

Experimental tests of BFKL predictions and finding of good observables for BFKL effects are very important for studying of the new high-energy domain of QCD.

The first LL BFKL calculations were applied for heavy quark production in photon collisions, ${ }^{9}$ deep inelastic lepton-nucleon scattering, ${ }^{44}$ heavy lepton-pair production $^{45}$ and high $p_{T}$ hadron production ${ }^{46-48}$ in hadronic collisions (see for a review Ref. 49).

Proposal ${ }^{50}$ to study the most forward and most backward in rapidity jets (Mueller-Navelet jets) in hadronic collisions was very promising since the BFKL effects could be very pronounced with increasing of the rapidity separation between Mueller-Navelet jets. Mueller-Navelet dijets is a special case of inclusive dijets, ${ }^{51}$ the latter are more suitable for jet tagging in limited rapidity coverage detectors. However, K-factor for Mueller-Navelet dijet production, ${ }^{50}$ which is ratio of the MuellerNavelet dijet cross section to Born cross section of two jet production, can not be experimentally measured. This is because Born cross section is purely theoretical quantity: one cannot forbid by experimental conditions virtual corrections to Born process.

So, there were suggested other jet observables: dijet azimuthal angle decorrelations, ${ }^{51-55}$ single jet ratio at different collision energies, ${ }^{56}$ dijet ratio at different collision energies, ${ }^{57}$ etc. In Refs. 58 and 59 there was proposed an analog of dijet K-factor: instead of Born dijet cross section one can use cross section of events when there are only two jets above $p_{T \text { min }}\left(p_{T \text { min-veto on extra jets). }}\right.$

At Fermilab Tevatron D $\emptyset$ Collaboration has measured for Mueller-Navelet dijets azimuthal angle decorrelations ${ }^{60}$ and ratio of Mueller-Navelet dijet cross sections 
at $1800 \mathrm{GeV}$ and $630 \mathrm{GeV}$ collisions energies. ${ }^{61}$ Unfortunately, those two measurements for Mueller-Navelet dijets are somewhat not consistent: the first one ${ }^{60}$ shows no indication of BFKL evolution manifestation, while the second one ${ }^{61}$ shows an overwhelming BFKL-like contribution.

The dijet "K-factor" as a function of rapidity separation was measured at LHC Run I at $7 \mathrm{TeV}$ by ATLAS (inverse "K-factor") ${ }^{62}$ and CMS. ${ }^{63}$ The ATLAS measurement ${ }^{62}$ had more limited than $\mathrm{CMS}$ one ${ }^{63}$ range in in rapidity separation and $p_{\text {Tmin }}$ (ATLAS: $\Delta y<6$ and $p_{\text {Tmin }}>70 \mathrm{GeV}$ versus CMS: $\Delta y<9.4$ and $\left.p_{\text {Tmin }}>35 \mathrm{GeV}\right)$.

In Fig. 5 it is shown a comparison of CMS data ${ }^{63}$ with various Monte Carlo (MC) event generators.

LL BFKL based MC generators CASCADE ${ }^{64}$ and HEJ+ARIADNE ${ }^{65}$ overestimate the CMS data. ${ }^{63}$ The MC generators based LL BFKL, which is designed for infinite energy, inherit its drawbacks at finite energy. So, this is not a strong surprise that LL BFKL based MC generators do not describe the data.

LL GLAPD based MC generator: HERWIG $++{ }^{66}$ overshoots the data, while LL GLAPD PYTHIA ${ }^{67}$ and $8^{68}$ provide a pretty good agreement with the data. However, the both HERWIG++ and PYTHIA generators contain beyond GLAPD contributions, such as color coherence, polar angle ordering, color dipole approximation, etc. Those contributions are small corrections in GLAPD kinematical domain

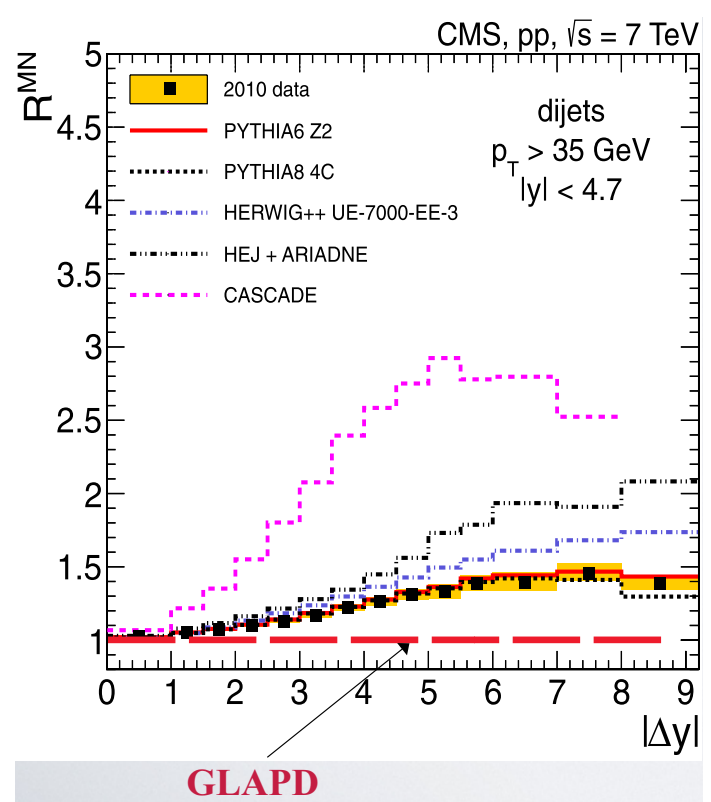

Fig. 5. Ratio of Mueller-Navelet dijet production cross section over two-jet cross section (dijet "K-factor") as a function of rapidity separation between jets at the LHC: CMS data at $7 \mathrm{TeV}$ versus prediction of GLAPD event generators with LL parton shower improved by beyond-GLAPD effects such as color coherence, color dipole approximation, etc. 
and often improve quality of data description. However, in BFKL domain those corrections are no longer valid, one has to use a proper tool: BFKL approach. One can see it in Fig. 5, instead of constant both LL GLAPD based MC generators HERWIG ++ and PYTHIA yield a noticeable rise with increasing of rapidity separation. Different implementations of those corrections provide a quite different behavior for HERWIG++ and PYTHIA. Unfortunately, there is no neither NLL BFKL generator nor pure GLAPD generator to confront with the data. So, one can check whether the description of the data by PYTHIA generators is accidental or not by testing other observables.

Indeed, preliminary CMS data ${ }^{69}$ for azimuthal angle decorrelations of dijets as a function of rapidity separation between jets show better overall description by HERWIG ++ than PYTHIA. However, the best description provide analytical NLL BFKL calculations, ${ }^{70}$ improved by BFKLP. ${ }^{12}$

Further progress for experimental tests of BFKL predictions will be related with LHC Run II and with development of BFKL MC generators by inclusion the NLL BFKL corrections.

\section{Summary and Outlooks}

The BFKLP approach, ${ }^{12}$ the generalized BLM scale setting for non-Abelian case, open new windows for applications of NLL BFKL evolution to the high-energy phenomenology.

Highly virtual photon-photon collisions provide a very unique opportunity to test high-energy asymptotics of QCD. The NLL BFKL predictions ${ }^{25,41}$ for the $\gamma^{*} \gamma^{*}$ total cross section with LO photon impact factors and with the renormalization scale fixed by BFKLP approach ${ }^{12}$ show a good agreement with the data from OPAL ${ }^{36}$ and L3 ${ }^{37}$ Collaborations at CERN LEP2. This is at the moment the most pronounced BFKL evolution manifestation in high energy phenomenology. Forthcoming complete NLL BFKL calculations for highly virtual photon collisions will shed light on the role of different ingredients of NLL BFKL approach.

The LHC data at $7 \mathrm{TeV}^{69}$ on dijet production with wide rapidity separation between jets are in a good agreement with analytical NLL BFKL calculations ${ }^{70}$ improved by BFKLP. ${ }^{12}$ Further progress for experimental tests of BFKL predictions for jet production will be related with recently started LHC Run II and finding new observables for BFKL evolution manifestation. The progress requires also a great development problem for BFKL MC generators by inclusion the NLL BFKL corrections.

\section{Acknowledgments}

V. T. K. is grateful to the organizers of the Hadron Structure Conference (HS'15) for their warm hospitality. This work is supported by the Russian Science Foundation, grant No. 14-22-00281. 


\section{References}

1. V. N. Gribov and L. N. Lipatov, Sov. J. Nucl. Phys. 15, 438 (1972), [Yad. Fiz. 15, $781(1972)]$.

2. V. N. Gribov and L. N. Lipatov, Sov. J. Nucl. Phys. 15, 675 (1972), [Yad. Fiz. 15, 1218 (1972)].

3. L. N. Lipatov, Sov. J. Nucl. Phys. 20, 94 (1975), [Yad. Fiz. 20, 181 (1974)].

4. G. Altarelli, G. Parisi, Nucl. Phys. B 126, 298 (1977).

5. Y. L. Dokshitzer, Sov. Phys. JETP 46, 641 (1977), [Zh. Eksp. Teor. Fiz. 73, 1216 (1977)].

6. V. S. Fadin, E. A. Kuraev and L. N. Lipatov, Phys. Lett. B 60, 50 (1975).

7. E. A. Kuraev, L. N. Lipatov and V. S. Fadin, Sov. Phys. JETP 44, 443 (1976), [Zh. Eksp. Teor. Fiz. 71, 840 (1976)].

8. E. A. Kuraev, L. N. Lipatov and V. S. Fadin, Sov. Phys. JETP 45, 199 (1977), [Zh. Eksp. Teor. Fiz. 72, 377 (1977)].

9. I. I. Balitsky and L. N. Lipatov, Sov. J. Nucl. Phys. 28, 822 (1978), [Yad. Fiz. 28, 1597 (1978)].

10. V. Fadin and L. Lipatov, Phys. Lett. B 429, 127 (1998).

11. M. Ciafaloni and G. Camici, Phys. Lett. B 430, 349 (1998).

12. S. Brodsky, V. Fadin, V. Kim, L. Lipatov and G. Pivovarov, JETP Lett. 70, 155 (1999).

13. V. S. Fadin, V. T. Kim, L. N. Lipatov and G. B. Pivovarov, The BFKL pomeron within physical renormalization schemes and scales, in Proc. of the 35th PNPI Winter School on Nuclear and Particle Physics, St. Petersburg, Repino, Russia, February 19-25, 2001, arXiv:hep-ph/0207296.

14. S. J. Brodsky, G. P. Lepage and P. B. Mackenzie, Phys. Rev. D 28, 228 (1983).

15. W. Celmaster and R. J. Gonsalves, Phys. Rev. Lett. 42, 1435 (1979).

16. W. Celmaster and R. J. Gonsalves, Phys. Rev. D 20, 1420 (1979).

17. L. N. Lipatov, Sov. Phys. JETP 63, 904 (1986), [Zh. Eksp. Teor. Fiz. 90, 1536 (1986)].

18. S. V. Mikhailov, JHEP 06, 009 (2007).

19. A. L. Kataev and S. V. Mikhailov, Theor. Math. Phys. 170, 139 (2012), [Teor. Mat. Fiz. 170, 174 (2012)].

20. A. L. Kataev and S. V. Mikhailov, Phys. Rev. D 91, 014007 (2015).

21. S. J. Brodsky and X.-G. Wu, Phys. Rev. D 85, 034038 (2012), [Erratum: Phys. Rev. D 86, 079903 (2012)].

22. S. J. Brodsky and L. Di Giustino, Phys. Rev. D 86, 085026 (2012).

23. M. Mojaza, S. J. Brodsky and X.-G. Wu, Phys. Rev. Lett. 110, 192001 (2013).

24. S. J. Brodsky, M. Mojaza and X.-G. Wu, Phys. Rev. D 89, 014027 (2014).

25. S. J. Brodsky, V. S. Fadin, V. T. Kim, L. N. Lipatov and G. B. Pivovarov, JETP Lett. 76, 249 (2002), [Pisma Zh. Eksp. Teor. Fiz. 76, 306 (2002)].

26. J. Bartels, A. De Roeck and H. Lotter, Phys. Lett. B 389, 742 (1996).

27. S. J. Brodsky, F. Hautmann and D. E. Soper, Phys. Rev. Lett. 78, 803 (1997), [Erratum: Phys. Rev. Lett. 79, 3544(1997)].

28. S. J. Brodsky, F. Hautmann and D. E. Soper, Phys. Rev. D 56, 6957 (1997).

29. A. Bialas, W. Czyz and W. Florkowski, Eur. Phys. J. C 2, 683 (1998).

30. M. Boonekamp, A. De Roeck, C. Royon and S. Wallon, Nucl. Phys. B 555, 540 (1999).

31. V. P. Goncalves, M. S. Kugeratski, E. R. Cazaroto, F. Carvalho and F. S. Navarra, Eur. Phys. J. C 71, 1779 (2011).

32. G. V. Frolov, V. N. Gribov and L. N. Lipatov, Phys. Lett. B 31, 34 (1970).

33. V. N. Gribov, L. N. Lipatov and G. V. Frolov, Sov. J. Nucl. Phys. 12, 543 (1971), [Yad. Fiz. 12, 994(1970)]. 
34. H. Cheng and T. T. Wu, Phys. Rev. D 1, 2775 (1970).

35. V. M. Budnev, I. F. Ginzburg, G. V. Meledin and V. G. Serbo, Phys. Rept. C 15, 181 (1975).

36. OPAL Collaboration, G. Abbiendi et al., Eur. Phys. J. C 24, 17 (2002).

37. L3 Collaboration, P. Achard et al., Phys. Lett. B 531, 39 (2002).

38. J. Bartels and A. Kyrieleis, Phys. Rev. D 70, 114003 (2004).

39. I. Balitsky and G. A. Chirilli, Phys. Rev. D 83, 031502 (2011).

40. I. Balitsky and G. A. Chirilli, Phys. Rev. D 87, 014013 (2013).

41. F. Caporale, D. Yu. Ivanov and A. Papa, Eur. Phys. J. C 58, 1 (2008).

42. J. Bartels and M. Lublinsky, JHEP 09, 076 (2003).

43. M. Cacciari, V. Del Duca, S. Frixione and Z. Trocsanyi, JHEP 02, 029 (2001).

44. L. V. Gribov, E. M. Levin and M. G. Ryskin, Nucl. Phys. B 188, 555 (1981).

45. E. M. Levin and M. G. Ryskin, Yad. Fiz. 32, 802 (1980).

46. M. G. Ryskin, Yad. Fiz. 32, 259 (1980).

47. L. V. Gribov, E. M. Levin and M. G. Ryskin, Phys. Lett. B 100, 173 (1981).

48. L. V. Gribov, E. M. Levin and M. G. Ryskin, Phys. Lett. B 121, 65 (1983).

49. L. V. Gribov, E. M. Levin and M. G. Ryskin, Phys. Rept. C 100, 1 (1983).

50. A. H. Mueller and H. Navelet, Nucl. Phys. B 282, 727 (1987).

51. V. T. Kim and G. B. Pivovarov, Phys. Rev. D 53, 6 (1996).

52. V. Del Duca and C. R. Schmidt, Phys. Rev. D 49, 4510 (1994).

53. W. J. Stirling, Nucl. Phys. B 423, 56 (1994).

54. A. Sabio Vera, Nucl. Phys. B 746, 1 (2006).

55. A. Sabio Vera and F. Schwennsen, Nucl. Phys. B 776, 170 (2007).

56. V. T. Kim and G. B. Pivovarov, Phys. Rev. D 57, 1341 (1998).

57. L. H. Orr and W. J. Stirling, Phys. Lett. B 429, 135 (1998).

58. V. B. Gavrilov, V. T. Kim, A. A. Krokhotin, G. B. Pivovarov and G. B. Safronov, Asymptotic effects in jet production at high energies, in Proc. of 15th International Seminar on High Energy Physics (QUARKS-2008), arXiv:hep-ph0901.1985.

59. V. B. Gavrilov, V. T. Kim, V. A. Murzin, V. A. Oreshkin, G. B. Pivovarov, I. Yu. Pozdnyakov and G. B. Safronov, Nucl. Phys. Proc. Suppl. 245, 153 (2013).

60. D0 Collaboration, S. Abachi et al., Phys. Rev. Lett. 77, 595 (1996).

61. D0 Collaboration, S. Abbott et al., Phys. Rev. Lett. 84, 5722 (2000).

62. ATLAS Collaboration, G. Aad et al., JHEP 1109, 053 (2011).

63. CMS Collaboration, S. Chatrchyan et al., Eur. Phys. J. C 72, 2216 (2012).

64. H. Jung, S. Baranov, M. Deak, A. Grebenyuk, F. Hautmann et al., Eur. Phys. J. C 70, 1237 (2010).

65. J. R. Andersen, L. Lonnblad and J. M. Smillie, JHEP 07, 110 (2011).

66. M. Bahr et al., Eur. Phys. J. C 58, 639 (2008).

67. T. Sjöstrand, S. Mrenna and P. Z. Skands, JHEP 05, 026 (2006).

68. T. Sjöstrand, S. Mrenna and P. Z. Skands, Comput. Phys. Commun. 178, 852 (2008).

69. C. Collaboration, CMS-PAS-FSQ-12-002, CERN (2013).

70. B. Duclou, L. Szymanowski and S. Wallon, Phys. Rev. Lett. 112, 082003 (2014). 